\title{
HMOX-1 inhibits TGF- $\beta$-induced epithelial-mesenchymal transition in the MCF-7 breast cancer cell line
}

\author{
XIAOFENG ZHU ${ }^{1 *}$, SHUICHUAN HUANG ${ }^{2 *}$, LILI ZENG $^{3}$, JIEYI MA $^{4}$, SUHONG SUN $^{1}$, \\ FENG ZENG $^{1}$, FANLI KONG ${ }^{1}$ and XIAOMING CHENG ${ }^{1}$
}

\author{
${ }^{1}$ Department of Breast and Thyroid Surgery, The Affiliated Hospital, Zunyi Medical College, Zunyi, Guizhou 563003; \\ ${ }^{2}$ Department of Vascular Surgery, Nanfang Hospital, Southern Medical University, Guangzhou, Guangdong 510515; \\ ${ }^{3}$ Department of Pathology, The Affiliated Hospital, Zunyi Medical College, Zunyi, Guizhou 563003; ${ }^{4}$ Laboratory of General Surgery, \\ The First Affiliated Hospital, Sun Yat-sen University, Guangzhou, Guangdong 510000, P.R. China
}

Received December 5, 2016; Accepted May 29, 2017

DOI: $10.3892 / \mathrm{ijmm} .2017 .3027$

\begin{abstract}
Epithelial-mesenchymal transition (EMT) is a key mechanism underlying metastatic breast cancer. Reactive oxygen species (ROS) play an important role in EMT. Heme oxygenase-1 (HMOX-1) can reduce oxidative stress. However, the effect of HMOX-1 on the EMT process in breast cancer cells is unknown. We treated the MCF-7 breast cancer cell line with the HMOX-1 inducer hemin and observed that hemin induced HMOX-1 expression and inhibited migration, invasion and ROS generation in transforming growth factor- $\beta$ (TGF- $\beta$ )-treated MCF-7 cells using quantitative RT-qPCR, western blotting, wound-healing and cell invasion assays as well as fluorescent probe DCFDA. Hemin inhibited TGF- $\beta$-induced EMT in the MCF-7 cells, whereas HMOX-1 siRNA attenuated the suppressive effect of hemin as determined by the expression and cellular distribution of selected EMT markers. In summary, our results revealed that hemin treatment increased HMOX-1 expression and inhibited TGF- $\beta$-induced EMT in MCF-7 cells.
\end{abstract}

\section{Introduction}

Tumor metastasis is a major clinical challenge that accounts for the vast majority of cancer-related deaths. Breast cancer patients have high rates of development of metastatic disease even following successful primary tumor resection and adjuvant therapy. An estimated 30\% of node-negative breast cancer

Correspondence to: Dr Xiaofeng Zhu or Dr Xiaoming Cheng, Department of Breast and Thyroid Surgery, The Affiliated Hospital, Zunyi Medical College, 72 Dalian Road, Zunyi, Guizhou 563003, P.R. China

E-mail: zhuxfshiwo@163.com

E-mail:cxm1688@sina.com

*Contributed equally

Key words: heme oxygenase-1, hemin, transforming growth factor- $\beta$, epithelial-mesenchymal transition, breast cancer patients and a larger percentage of node-positive breast cancer patients develop metastatic disease despite receiving standard therapy (1). Approximately $90 \%$ of breast cancer deaths are caused by the local invasion and distant metastasis of tumor cells (2). The path of metastatic colonization is a complex and multi-faceted process. To develop metastasis, primary cancer cells must invade and escape the physical barriers at the primary site.

Epithelial-mesenchymal transition (EMT) is a process whereby cancer cells lose their epithelial properties to acquire a mesenchymal phenotype and become motile and invasive, which is linked to metastasis (3-5). The expression of the intercellular epithelial adhesion molecule E-cadherin is decreased, and markers of mesenchymal cells, such as $\alpha$-smooth muscle actin ( $\alpha$-SMA), N-cadherin and vimentin, are upregulated during EMT (3). EMT has also been connected to the induction of cancer stem cells and drug resistance, suggesting that EMT may underlie many biological processes of cancer development (4). Transforming growth factor- $\beta$ (TGF- $\beta$ ) is secreted by many cell types and directly stimulates the production of the extracellular matrix and microenvironment by both normal and cancer cells (6). As tumors progress, TGF- $\beta$ induces neoplastic cell invasiveness and metastasis by promoting EMT in many cancer cell types (7). Reactive oxygen species (ROS) are radicals, ions or molecules that have a single unpaired electron in their outermost shell and are constantly generated inside cells by dedicated enzyme complexes or as by-products of redox reactions (8). A recent study suggested that ROS play an important role in TGF- $\beta$-induced EMT. A significant increase was found to occur in intracellular ROS upon TGF- $\beta$ stimulation (9). The release of TGF- $\beta$-dependent ROS is responsible for the phosphorylation of Smad2, p38 MAPK and extracellular signal-regulated kinase $1 / 2$ (ERK1/2) and accounts for the upregulation of $\alpha$-SMA and fibronectin and the repression of E-cadherin (9).

The cellular defense system against oxidative stress is composed of a subset of antioxidant proteins. Heme oxygenase-1 (HMOX-1) is a microsomal enzyme that is induced in response to cellular stress and diverse oxidative stimuli (10). The enzymatic activity of HMOX-1 produces CO, ferrous iron and biliverdin. Therefore, HMOX-1 can reduce oxidative 
stress, attenuate inflammatory responses and lower the rate of apoptosis (10). HMOX-1 can also inhibit the migration and invasion of prostate cancer cells and renal tubular epithelial cells (11). Additionally, the biological activity of HMOX-1 reduces ROS generation (10). However, the effect of HMOX-1 on EMT, which plays a critical role in the metastasis of breast cancer, requires further research.

In this study, we found that the HMOX-1 inducer hemin inhibited ROS production in the MCF-7 breast cancer cell line. Furthermore, we observed that hemin inhibited the migration, invasion and EMT of MCF-7 breast cancer cells. These results show that HMOX-1 may function as an important player in breast cancer metastasis.

\section{Materials and methods}

Cell culture. The MCF-7 human breast cancer cell line was purchased from the American Type Culture Collection (ATCC; Manassas, VA, USA) and cultured in Dulbecco's modified Eagle's medium (DMEM) supplemented with $10 \%$ fetal bovine serum (FBS), $1 \%$ penicillin and $1 \%$ streptomycin (both from Gibco, Karlsruhe, Germany) in a $5 \% \mathrm{CO}_{2}$ atmosphere at $37^{\circ} \mathrm{C}$.

Transfection of siRNAs. The HMOX-1 siRNA was synthesized by RiboBio Biotechnology (Guangzhou, China). The HMOX-1 siRNA sequences were sense, 5'-CCAGCAACAAAGUGCA AGAdTdT-3' and antisense, 3'-dTdTGGUCGUUGUUUCACG UUCU-5'. Breast cancer cells were transfected with $50 \mathrm{nM}$ of siRNA for $8 \mathrm{~h}$ using the RNAiMAX transfection agent (Invitrogen, Carlsbad, CA, USA) according to the manufacturer's instructions.

Reverse transcription-quantitative (real-time) polymerase chain reaction $(R T-q P C R)$ for $m R N A$ quantification. Total RNA was extracted from the cells using TRIzol (Invitrogen), and cDNA was synthesized from 1,000 ng of total RNA using the PrimeScript RT reagent kit (Takara, Dalian, China) following the manufacturer's instructions. Quantitative PCR was performed on the Bio-Rad CFX 96 real-time PCR machine (Bio-Rad Laboratories, Inc., Hercules, CA, USA) using SYBR ${ }^{\circledR}$ Premix Ex Taq ${ }^{\mathrm{TM}}$ II (Tli RNaseH Plus) (Takara). The primer sequences were as follows: $H M O X-1-\mathrm{F}, 5$-TACCACATCTAT GTGGCCCTG-3' and $H M O X-1$-R, 5'-TGGCTGGTGTGTA GGG GAT-3', and glyceraldehyde 3-phosphate dehydrogenase $(G A P D H)-\mathrm{F}, 5 '-G C A C C G T C A A G G C T G A G A A C-3 '$ and GAPDH-R, 5'-TGGTGAAGACGCCAGTGGA-3'. Data analysis was performed using the comparative $\mathrm{Ct}$ method with the Bio-Rad Manager 2.1 software (Bio-Rad Laboratories, Inc.).

Western blotting. The cells were lysed in RIPA lysis buffer (Cell Signaling Technology, Boston, MA, USA) supplemented with protease inhibitor (Roche, Basel, Switzerland). The total protein concentration was determined using a BCA kit (Keygen, Nanjing, China). Equal amounts of protein $(35 \mu \mathrm{g})$ for each group were separated by sodium dodecyl sulfate (SDS)-polyacrylamide gel electrophoresis, transferred to polyvinylidene difluoride (PVDF) membranes (Millipore, Bedford, MA, USA), and blotted using anti-HMOX-1 (ab52947; Abcam, Cambridge, UK), anti-E-cadherin (\#3195), anti-vimentin (\#5741) or anti- $\beta$-tubulin (\#2128) antibodies
(Cell Signaling Technology). The bands were visualized using the Luminol reagent (Thermo Pierce, Waltham, MA, USA) and imaged using the GE ImageQuant Las 4000 Mini (GE Healthcare, Fairfield, CT, USA).

Migration and invasion assays of MCF-7 breast cancer cells. The migratory ability of the MCF-7 cells was determined using a wound-healing assay. Briefly, MCF-7 cells were treated with hemin (20 $\mu \mathrm{M}$; Sigma-Aldrich, St. Louis, MO, USA) for $72 \mathrm{~h}$ and then seeded into 6-well plates $(60,000$ cells/well). When the cells were almost $100 \%$ confluent, the monolayer was wounded using a sterilized $200-\mu 1$ disposable pipette tip to scratch a wound in each well. Then, the cells were treated with TGF- $\beta 1$ (10 ng/ml; Peprotech, Rocky Hill, NJ, USA). The scratch wounds were visualized under a microscope (Zeiss Axio Observer Z1; Zeiss, Jena, Germany).

The cell invasion assay was performed in the BD BioCoat ${ }^{\mathrm{TM}}$ Matrigel $^{\mathrm{TM}}$ Invasion Chamber $(8-\mu \mathrm{m}$ pore size) (BD Bioscience, Franklin Lakes, NJ, USA). MCF-7 cells were treated with hemin $(20 \mu \mathrm{M})$ for $72 \mathrm{~h}$. Then, the cells were added to the upper chambers with $200 \mu \mathrm{l}$ of serum-free DMEM medium, and the lower chambers were filled with $500 \mu \mathrm{l}$ of DMEM medium supplemented with TGF- $\beta 1$ (10 ng/ $\mathrm{ml}$ ). After $12 \mathrm{~h}$, non-migrating cells were removed from the upper chamber, and the migrating cells adhering to the lower surface of the membrane were fixed with $4 \%$ formaldehyde and quantified by $0.1 \%$ crystal violet staining.

Immunofluorescence assay. The cells were seeded into 24-well plates and treated with TGF- $\beta 1(10 \mathrm{ng} / \mathrm{ml})$ for 5 days. The cells were washed in phosphate-buffered saline (PBS), fixed in $4 \%$ formaldehyde for $15 \mathrm{~min}$, permeabilized with $0.1 \%$ Triton X-100 for $10 \mathrm{~min}$, and blocked with $0.1 \%$ BSA for $1 \mathrm{~h}$. Then, the cells were incubated with an anti-E-cadherin (\#3195) or anti-vimentin (\#5741) antibody (Cell Signaling Technology) overnight at $4^{\circ} \mathrm{C}$. After washing with PBS 3 times, the cells were incubated with a fluorescent-conjugated secondary antibody (red, A11008; green, A11010; Life Technologies, Grand Island, NY, USA) for $1 \mathrm{~h}$ at room temperature and stained with 4',6-diamidino-2-phenylindole (DAPI; Roche) for $10 \mathrm{~min}$. Images were acquired using a fluorescence microscope (Carl Zeiss Axio Observer Z1, Jena, Germany).

Fluorescent ROS assay. ROS generation was analyzed after staining the cells with the fluorescent probe dichlorofluorescein-DA (DCFDA) (Sigma-Aldrich). The cells were incubated with or without hemin following TGF- $\beta 1$ treatment. Then, the cells were loaded with DCFDA (20 mM) in Hank's Balanced Salt Solution (HBSS; Gibco, Karlsruhe, Germany) at $37^{\circ} \mathrm{C}$ for $30 \mathrm{~min}$ in the dark. After washing with HBSS, the DCFDA fluorescence of each well was measured at $485 \mathrm{~nm}$ (excitation) and $528 \mathrm{~nm}$ (emission) with a Multi-Mode Microplate Reader (BioTek, Winooski, VT, USA).

Statistical analysis. The data are expressed as the mean \pm SD. Differences between the treatment groups and the control group were assessed with Student's t-test using GraphPad Prism version 5.0 (GraphPad, San Diego, CA, USA). Statistically significant differences are indicated as $\mathrm{P}<0.05, \mathrm{P}<0.01$ and $\mathrm{P}<0.001$. 
A

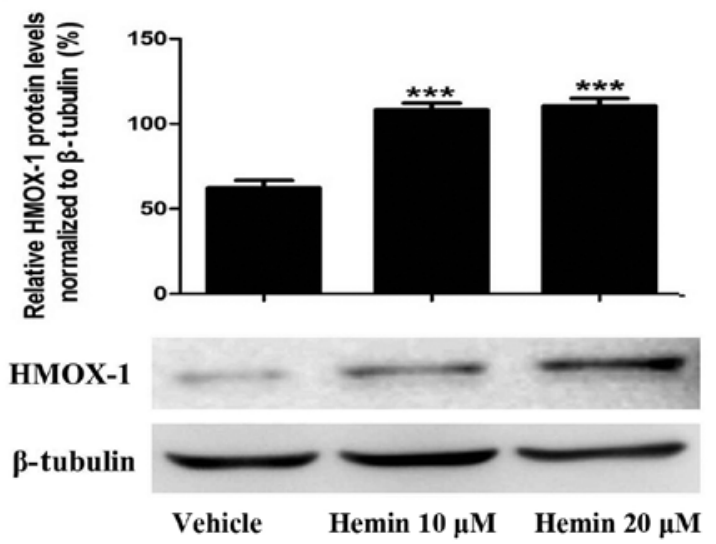

C

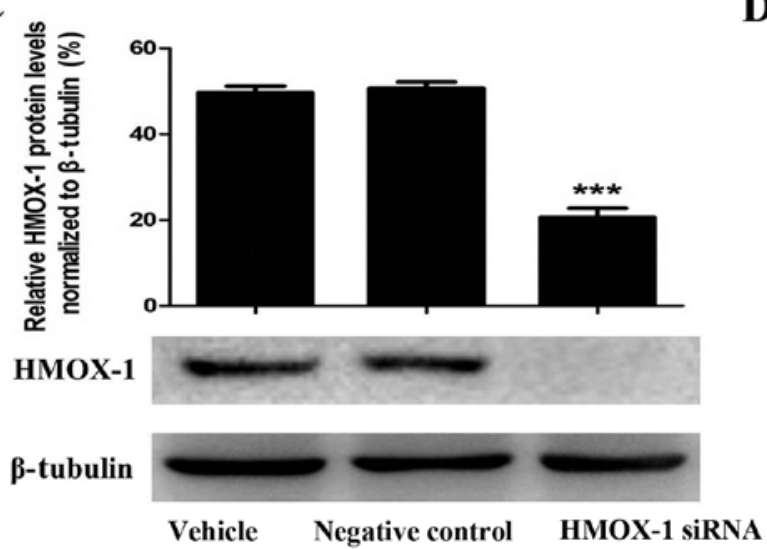

B

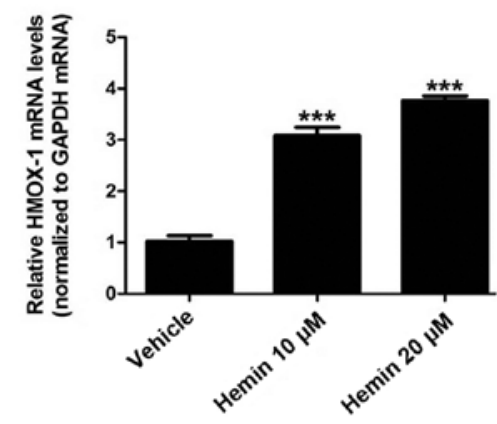

D

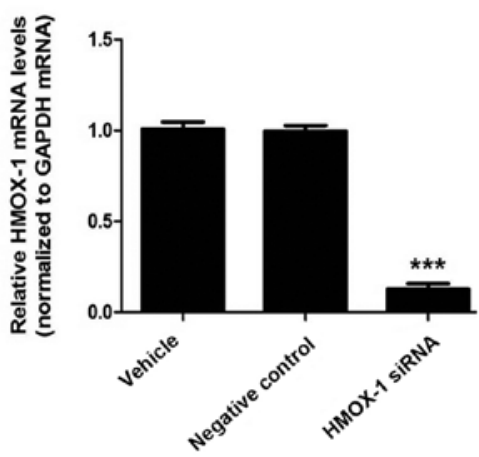

Figure 1. Hemin induces heme oxygenase-1 (HMOX-1) expression in the MCF-7 breast cancer cell line. (A) Western blotting showed that hemin (20 $\mu$ M, $72 \mathrm{~h}$ )-treated MCF-7 cells expressed a higher HMOX-1 protein level than that in the control cells. (B) qRT-PCR showed that hemin (20 $\mu$ M, 48 h) induced HMOX-1 mRNA expression in the MCF-7 cells. (C and D) Both western blotting and qRT-PCR showed that HMOX-1 siRNA inhibited HMOX-1 expression in the hemin-treated MCF-7 cells. All the experiments were repeated at least three times with similar results and one representative from at least three independent experiments is shown. The results are shown as the mean $\pm \mathrm{SD},{ }^{* * *} \mathrm{P}<0.001$, experimental vs. vehicle value.

\section{Results}

Hemin induces HMOX-1 expression in the MCF-7 breastcancer cell line. To evaluate the effect of HMOX-1 on TGF- $\beta$-induced EMT, we treated MCF-7 cells with an HMOX-1 inducer (hemin, $20 \mu \mathrm{M}$ ) and then examined whether hemin induced HMOX-1 expression using RT-qPCR and western blotting. Hemin treatment significantly upregulated HMOX-1 mRNA and protein expression in the MCF-7 cells (Fig. 1A and B). Then, we confirmed that the HMOX-1 siRNA knocked down hemin-induced HMOX-1 expression by RT-qPCR and western blotting (Fig. 1C and D).

Hemin inhibits the migration and invasion of TGF- $\beta$-treated MCF-7 cells. Wound-healing and cell invasion assays were performed to investigate the effect of HMOX-1 on the migration and invasion of TGF- $\beta 1$-treated MCF-7 cells after incubating the cells with or without $20 \mu \mathrm{M}$ of hemin. Hemin significantly inhibited the migratory ability of the MCF-7 cells in the wound-healing assay (Fig. 2A and B). Additionally, upregulation of HMOX-1 by hemin significantly inhibited the invasiveness of the MCF-7 cells (Fig. 2C and D). MCF-7 cells were treated with hemin $(20 \mu \mathrm{M})$ for $72 \mathrm{~h}$ and then transfected with the HMOX-1 siRNA or a negative control. Then, we induced the migration and invasion of the MCF-7 cells with TGF- $\beta 1(10 \mathrm{ng} / \mathrm{ml})$. The HMOX-1 siRNA attenuated the inhibitory effect of hemin on the MCF-7 cells and promoted the migration (Fig. 2E and F) and invasion (Fig. 2G and H) of the hemin-treated MCF-7 cells.

Hemin inhibits the TGF- $\beta$-induced EMT in MCF- 7 cells. The morphological changes characteristic of cells undergoing EMT are accompanied by a shift in gene expression from an epithelial to a mesenchymal repertoire. To determine whether HMOX-1 inhibits this shift, both the expression and cellular distribution of selected EMT markers were investigated by immunofluorescence staining and western blotting in the TGF- $\beta 1$-treated MCF-7 cells. The MCF-7 cells exhibited decreased E-cadherin expression and higher expression of the mesenchymal marker vimentin after TGF- $\beta 1$ treatment $(10 \mathrm{ng} / \mathrm{ml}$ ) (Fig. 3B-D). These changes were reversed by sequentially treating the cells with $20 \mu \mathrm{M}$ of hemin. These results implied that HMOX-1 inhibited the EMT progression in MCF-7 cells. Our data showed that HMOX-1 prevented EMT changes in the MCF-7 cells. To confirm this finding, we performed a loss-of-function study in which the HMOX-1 siRNA was used to verify the effect of HMOX-1 on EMT. After exposure to hemin $(20 \mu \mathrm{M})$ for 

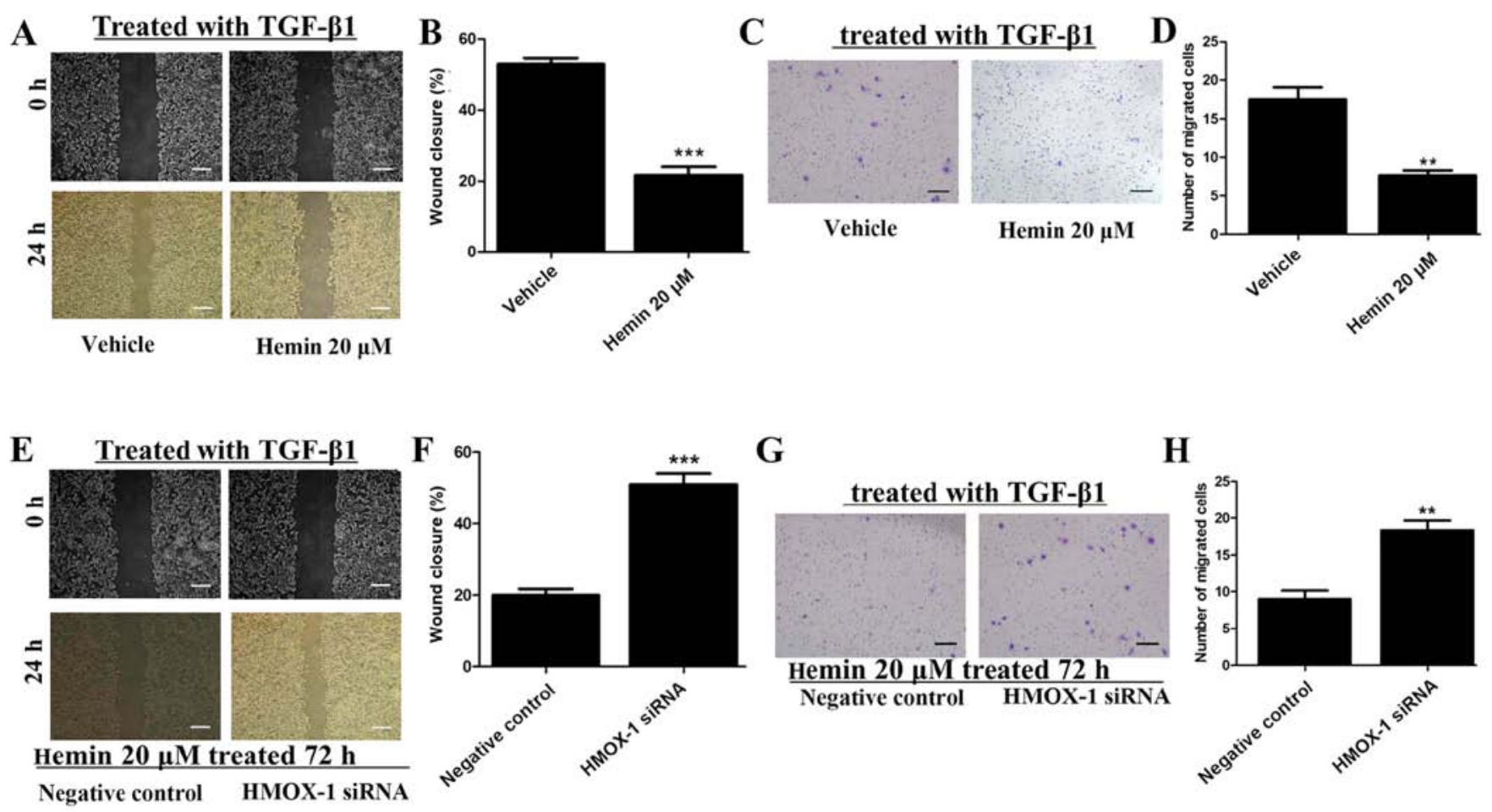

Figure 2. Hemin inhibits the migration and invasion of transforming growth factor- $\beta$ (TGF- $\beta$ )-treated MCF-7 cells. (A and B) MCF-7 cell were pretreated with hemin $(20 \mu \mathrm{M}, 72 \mathrm{~h})$ and subjected to the wound-healing assay. The results showed that hemin significantly decreased the migration of the TGF- $\beta 1$ (10 ng/ml)-induced MCF-7 cells. (C and D) Cancer cell invasion assay showed that hemin significantly decreased the invasive capacity of the TGF- $\beta 1$-treated MCF-7 cells. (E and F) A wound-healing assay showed that the heme oxygenase-1 (HMOX-1) siRNA significantly promoted the migration of the TGF- $\beta 1$-induced MCF-7 cells previously exposed to hemin $(20 \mu \mathrm{M}, 72 \mathrm{~h})$. (G and H) A cancer cell invasion assay showed that HMOX-1 siRNA significantly promoted the invasion of the TGF- $\beta 1$-induced MCF-7 cells previously exposed to hemin $(20 \mu \mathrm{m}, 72 \mathrm{~h})$. Scale bar, $200 \mu \mathrm{m}$. All the experiments were repeated at least for three times with similar results and one representative from at least three independent experiments is shown. The result are shown as the mean $\pm \mathrm{SD},{ }^{* * *} \mathrm{P}<0.01$ and ${ }^{* * * * *} \mathrm{P}<0.001$.

$72 \mathrm{~h}$, the cells were transfected with the HMOX-1 siRNA or a negative control RNA and then treated with TGF- $\beta 1$ $(10 \mathrm{ng} / \mathrm{ml})$ for 5 days and subjected to western blotting and immunofluorescence assays. The results of both the western blotting and immunofluorescence assays showed that hemin inhibited the TGF- $\beta$-induced EMT in MCF-7 cells through the upregulation of E-cadherin and downregulation of vimentin. In contrast, diminished E-cadherin expression and increased vimentin expression were observed in the HMOX-1 siRNA-treated cells compared to the vehicle group (Fig. 3F-H). These results indicated that the HMOX-1 siRNA attenuated the inhibitory effect of hemin on the TGF- $\beta$-induced EMT in the MCF-7 cells.

Hemin reduces $R O S$ generation and induces E-cadherin expression in MCF-7 cells. To determine the role of HMOX-1 as a TGF- $\beta$-responsive ROS inhibitor, we treated the cells with TGF- $\beta 1(20 \mathrm{ng} / \mathrm{ml})$. ROS generation was measured using DCFDA and a fluorescence spectrometer. The hemin-treated MCF-7 cells had diminished ROS production compared to the control cells (Fig. 4A). These results were confirmed by silencing HMOX-1 with the HMOX-1 siRNA. The HMOX-1 siRNA-transfected group showed increased TGF- $\beta 1$-mediated ROS generation compared to the negative control group (Fig. 4B). Our findings suggested that HMOX-1 regulated the ROS levels in MCF-7 cells. Interestingly, we observed that hemin induced $\mathrm{E}$-cadherin protein expression in the normal MCF-7 cells (Fig. 4C and D).

\section{Discussion}

Metastasis is responsible for $\sim 90 \%$ of cancer-associated patient mortality (2). Although a great deal of effort has been expended, progress in the research on metastasis is stagnating due to the lack of effective tools to elucidate the complex network of signaling pathways that drive this multistep process. EMT is a phenotypic conversion linked to metastasis that was originally defined as a morphological conversion occurring at specific sites in embryonic epithelia to give rise to individual migratory cells (3). Recent studies have found that EMT plays a role in enhancing the invasive and metastatic behaviors of tumor cells. During the EMT process, epithelial cells gain mesenchymal properties and exhibit reduced intercellular adhesion and increased motility. Sequentially, these cells break through the basal membrane and migrate long distances (4). In this study, we showed that HMOX-1 inhibited TGF- $\beta$-induced EMT in the MCF-7 breast cancer cell line.

HMOX-1 is an anti-inflammatory and antioxidant protein. The role of HMOX-1 in cancer is controversial. Because this protein is a potent inducer of vascular endothelial growth factor (VEGF), which is a crucial factor that governs tumor angiogenesis, HMOX-1 has also been recognized as an angiogenesis exciter and a tumor-metastasis supporter (12). Alternatively, HMOX-1 has been reported to suppress the invasive capacity of breast cancer cells via downregulating matrix metallopeptidase 9 (MMP9) (13). In this study, we 
A

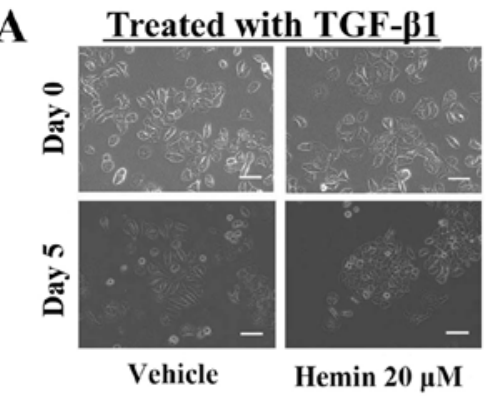

C

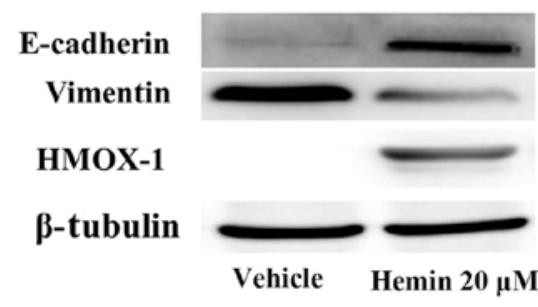

B

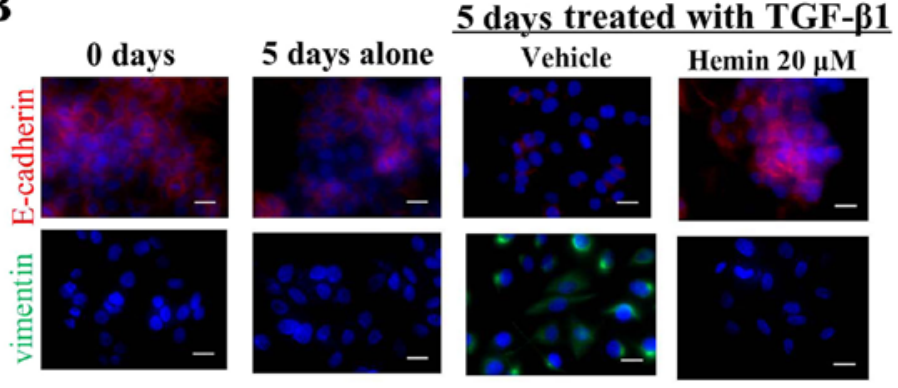

D

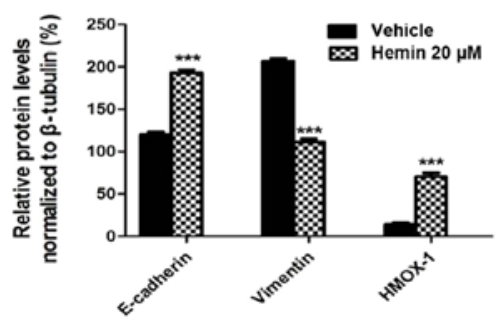

E

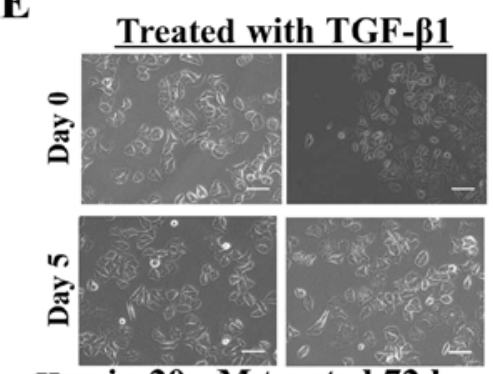

Hemin $20 \mu M$ treated $72 \mathrm{~h}$

Negative control HMOX-1 siRNA

G

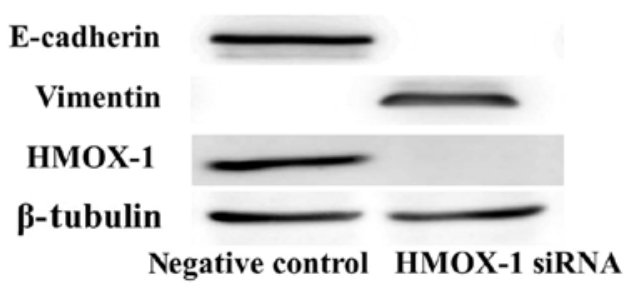

F

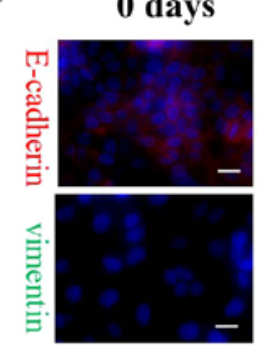

Hemin $20 \mu M$ treated $72 \mathrm{~h}$
H

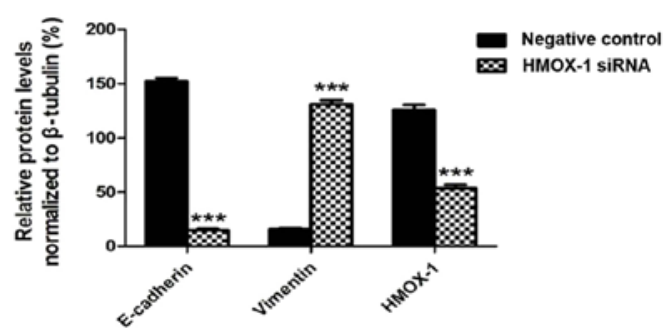

Figure 3. Hemin inhibits the transforming growth factor- $\beta 1$ (TGF- $\beta 1$ )-induced epithelial-mesenchymal transition (EMT) of the MCF-7 breast cancer cell line. MCF-7 cells were exposed to hemin $(20 \mu \mathrm{M}, 72 \mathrm{~h})$ or vehicle and then treated with TGF- $\beta$ (10 ng/ml) and subjected to the respective assays. (A) Representative photomicrograph showing that hemin inhibited the change in morphology from epithelial to mesenchymal. Scale bar, $100 \mu \mathrm{m}$. (B) E-cadherin (red) and vimentin (green) expression was visualized by immunofluorescence staining. 4',6-Diamidino-2-phenylindole (DAPI) (blue) was used for nuclear staining. Scale bar, $20 \mu \mathrm{m}$. (C and D) E-cadherin and vimentin protein expression was analyzed by western blotting. MCF-7 cells were exposed to hemin ( $20 \mu \mathrm{M}, 72 \mathrm{~h})$, transfected with the heme oxygenase-1 (HMOX-1) siRNA or negative control, and then treated with TGF- $\beta$ (10 ng/ml) and subjected to the respective assays. (E) Representative photomicrograph showing the cell morphology. Scale bar, $100 \mu \mathrm{m}$. (F) E-cadherin (red) and vimentin (green) expression was visualized by immunofluorescence staining. DAPI (blue) was used for nuclear staining. Scale bar, $20 \mu \mathrm{m}$. (G and $\mathrm{H}$ ) E-cadherin and vimentin protein expression was analyzed by western blotting. All the experiments were repeated at least three times with similar results and one representative from at least three independent experiments is shown. The results are shown as the mean $\pm \mathrm{SD},{ }^{* * * *} \mathrm{P}<0.001$.

found that HMOX-1 was induced by hemin in the breast cancer MCF-7 cell line and inhibited the invasion and migration of TGF- $\beta$-treated MCF-7 breast cancer cells.

TGF- $\beta$ is a primary inducer of EMT and plays a double role in cancer. TGF- $\beta$ inhibits the proliferation and induces the apoptosis of cancer cells in the early stages of tumorigenesis. However, this protein can also promote invasion and metastasis during later tumor development (7). TGF- $\beta$ induces
EMT by activating E-cadherin repressors and Smad2/3 (14). Additionally, TGF- $\beta$ can induce EMT via Smad-independent pathways, such as the phosphatidylinositol 3-kinase (PI3K), Akt, mitogen-activated protein kinase (MAPK) and Rho family GTPase pathways (15). Our data suggest a tumorsuppressor role for HMOX-1 in breast cancer. HMOX-1 expression was increased via hemin stimulation, and in turn the EMT induced by TGF- $\beta$ was inhibited in the hemin-treated 
A

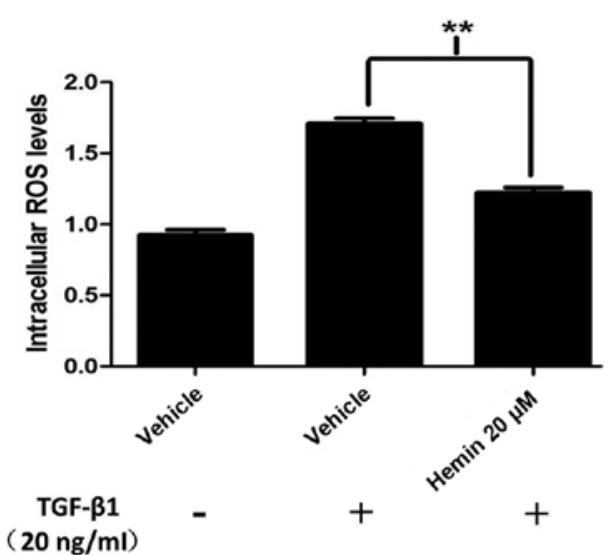

C

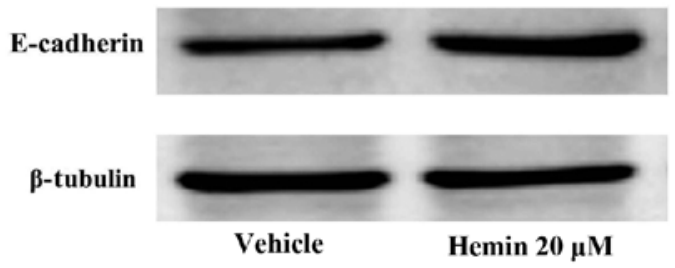

B

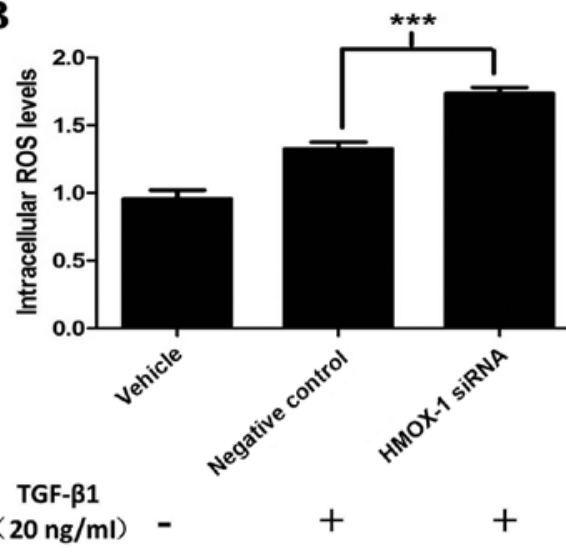

D

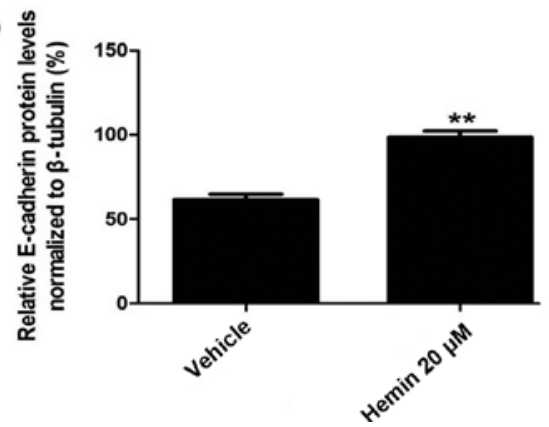

Figure 4. Heme oxygenase-1 (HMOX-1) decreases reactive oxygen species (ROS) generation and induces E-cadherin expression in MCF-7 cells. (A) MCF-7 cells were treated with hemin $(20 \mu \mathrm{M})$ or vehicle for $72 \mathrm{~h}$, pretreated with dichlorofluorescein-DA (DCFDA) (20 $\mu \mathrm{M})$ for $1 \mathrm{~h}$ and then incubated with or without transforming growth factor- $\beta$ (TGF- $\beta)(20 \mathrm{ng} / \mathrm{ml})$ for $24 \mathrm{~h}$. Then, the cells were lysed and centrifuged at $16,000 \mathrm{rpm}$ for $10 \mathrm{~min}$ and the cell supernatants were collected. ROS generation was measured at $485 \mathrm{~nm}$ (excitation) and $528 \mathrm{~nm}$ (emission) with a multi-mode microplate reader. (B) MCF-7 cells were treated with hemin $(20 \mu \mathrm{M})$ for $72 \mathrm{~h}$, transfected with the HMOX-1 siRNA or negative control, pretreated with DCFDA (20 $\mu \mathrm{M})$ for $1 \mathrm{~h}$ and then incubated with or without TGF- $\beta(20 \mathrm{ng} / \mathrm{ml})$ for $24 \mathrm{~h}$. ROS generation was measured at $485 \mathrm{~nm}$ (excitation) and $528 \mathrm{~nm}$ (emission) by a multi-mode microplate reader. (C and D) Western blot analyses showing that hemin $(20 \mu \mathrm{M}, 24 \mathrm{~h})$-treated MCF-7 cells expressed a higher E-cadherin protein level than that in the control cells. All the experiments were repeated at least three times with similar results and one representative from at least three independent experiments is shown. The results are shown as the mean $\pm \mathrm{SD},{ }^{* *} \mathrm{P}<0.01$ and ${ }^{* * *} \mathrm{P}<0.001$.

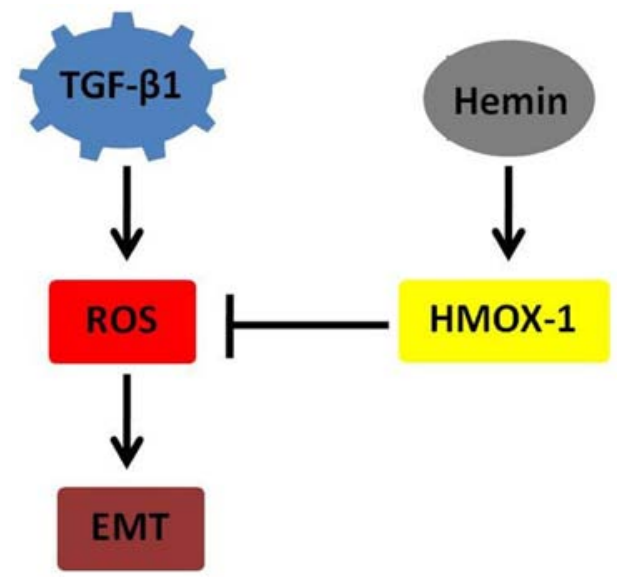

Figure 5. Hemin induces the expression of heme oxygenase-1 (HMOX-1), which inhibits transforming growth factor- $\beta 1$ (TGF- $\beta 1$ )-induced epithelialmesenchymal transition (EMT) via downregulating the generation of reactive oxygen species (ROS).

breast cancer cells. The inhibitory effect of hemin on the TGF- $\beta$-induced EMT was significantly attenuated by transfection of the HMOX-1 siRNA into hemin-treated MCF-7 breast cancer cells.
Recent observations suggest that ROS play an important role in TGF- $\beta$-induced EMT (16-18). Upon TGF- $\beta$ stimulation, a significant increase was found to occur in intracellular ROS. TGF- $\beta$-dependent ROS release was demonstrated to be responsible for the phosphorylation of $\operatorname{Smad} 2$ and p38 MAPK, the upregulation of $\alpha$-SMA and fibronectin and the downregulation of E-cadherin (13). Therefore, HMOX-1 may impact the EMT process by regulating the release of ROS and their dependent pathways (Fig. 5). Thus, modulating HMOX-1 levels may be a strategy through which to regulate TGF- $\beta$-induced EMT.

Interestingly, we found that HMOX-1 enhanced E-cadherin expression in normal MCF-7 cells. Epithelial cells communicate with their neighboring cells through the extension of either lamellipodia or filopodia, which couple to the intracellular cytoskeleton, trigger specific signaling pathways, and contribute to cell adhesion $(19,20)$. E-cadherin plays a major role in intercellular adhesion. Additionally, the role of E-cadherin as a tumor suppressor has been observed in a variety of cancers, including prostate, ovarian, gastric and breast cancers (21-24). Cancer cells with low E-cadherin expression levels have been suggested to be more invasive, although the signaling pathways involved in this modulation are unknown. Future study should focus on the potential 
molecular partners with which HMOX-1 interacts to induce the cellular morphological changes.

In conclusion, we found that HMOX-1 inhibited TGF- $\beta$-induced EMT by regulating ROS release in MCF-7 cells. Our results imply that HMOX-1 plays an antitumor role in the breast and acts as a target for cancer therapy.

\section{Acknowledgements}

This study was supported by the National Natural Science Foundation of China (no. 81172337).

\section{References}

1. Libson S and Lippman M: A review of clinical aspects of breast cancer. Int Rev Psychiatry 26: 4-15, 2014.

2. Criscitiello C, André F, Thompson AM, De Laurentiis M, Esposito A, Gelao L, Fumagalli L, Locatelli M, Minchella I, Orsi $\mathrm{F}$, et al: Biopsy confirmation of metastatic sites in breast cancer patients: Clinical impact and future perspectives. Breast Cancer Res 16: 205, 2014

3. De Craene B and Berx G: Regulatory networks defining EMT during cancer initiation and progression. Nat Rev Cancer 13: 97-110, 2013.

4. Sharma RR, Pollock K, Hubel A and McKenna D: Mesenchymal stem or stromal cells: A review of clinical applications and manufacturing practices. Transfusion 54: 1418-1437, 2014.

5. Kupcova Skalnikova H: Proteomic techniques for characterisation of mesenchymal stem cell secretome. Biochimie 95 2196-2211, 2013

6. Akhurst RJ and Hata A: Targeting the TGF $\beta$ signalling pathway in disease. Nat Rev Drug Discov 11: 790-811, 2012.

7. Li B, Wen G, Zhao Y, Tong J and Hei TK: The role of TGFBI in mesothelioma and breast cancer: Association with tumor suppression. BMC Cancer 12: 239, 2012.

8. Brown DI and Griendling KK: Regulation of signal transduction by reactive oxygen species in the cardiovascular system. Circ Res 116: 531-549, 2015.

9. Krstić J, Trivanović D, Mojsilović S and Santibanez JF: Transforming growth factor-beta and oxidative stress interplay: Implications in tumorigenesis and cancer progression, Oxid Med Cell Longev 2015: 654594, 2015.

10. Jozkowicz A, Was H and Dulak J: Heme oxygenase-1 in tumors: Is it a false friend? Antioxid Redox Signal 9: 2099-2117, 2007.

11. Gueron G, Giudice J, Valacco P, Paez A, Elguero B, Toscani M, Jaworski F, Leskow FC, Cotignola J, Marti M, et al: Heme-oxygenase-1 implications in cell morphology and the adhesive behavior of prostate cancer cells. Oncotarget 5: 4087-4102, 2014.
12. Cherrington JM, Strawn LM and Shawver LK: New paradigms for the treatment of cancer: The role of anti-angiogenesis agents. Adv Cancer Res 79: 1-38, 2000.

13. Chen HW, Chao CY, Lin LL, Lu CY, Liu KL, Lii CK and Li CC: Inhibition of matrix metalloproteinase-9 expression by docosahexaenoic acid mediated by heme oxygenase 1 in 12-O-tetradecanoylphorbol-13-acetate-induced MCF-7 human breast cancer cells. Arch Toxicol 87: 857-869, 2013.

14. Zavadil J, Cermak L, Soto-Nieves N and Böttinger EP: Integration of TGF-beta/Smad and Jagged1/Notch signalling in epithelialto-mesenchymal transition. EMBO J 23: 1155-1165, 2004.

15. Vincent T, Neve EP, Johnson JR, Kukalev A, Rojo F, Albanell J, Pietras K, Virtanen I, Philipson L, Leopold PL, et al: A SNAIL1-SMAD3/4 transcriptional repressor complex promotes TGF-beta mediated epithelial-mesenchymal transition. Nat Cell Biol 11: 943-950, 2009.

16. Zhang KH, Tian HY, Gao X, Lei WW, Hu Y, Wang DM, Pan XC, Yu ML, Xu GJ, Zhao FK, et al: Ferritin heavy chain-mediated iron homeostasis and subsequent increased reactive oxygen species production are essential for epithelial-mesenchymal transition. Cancer Res 69: 5340-5348, 2009.

17. Lim SO, Gu JM, Kim MS, Kim HS, Park YN, Park CK, Cho JW, Park YM and Jung G: Epigenetic changes induced by reactive oxygen species in hepatocellular carcinoma: Methylation of the E-cadherin promoter. Gastroenterology 135: 2128-2140, 2140.e1-2140.e8, 2008

18. Lochter A, Galosy S, Muschler J, Freedman N, Werb Z and Bissell MJ: Matrix metalloproteinase stromelysin-1 triggers a cascade of molecular alterations that leads to stable epithelialto-mesenchymal conversion and a premalignant phenotype in mammary epithelial cells. J Cell Biol 139: 1861-1872, 1997.

19. Baum B and Georgiou M: Dynamics of adherens junctions in epithelial establishment, maintenance, and remodeling. J Cell Biol 192: 907-917, 2011

20. Herszterg S, Leibfried A, Bosveld F, Martin C and Bellaiche Y: Interplay between the dividing cell and its neighbors regulates adherens junction formation during cytokinesis in epithelial tissue. Dev Cell 24: 256-270, 2013.

21. Tomita K, van Bokhoven A, van Leenders GJ, Ruijter ET, Jansen CF, Bussemakers MJ and Schalken JA: Cadherin switching in human prostate cancer progression. Cancer Res 60: 3650-3654, 2000.

22. Sawada K, Mitra AK, Radjabi AR, Bhaskar V, Kistner EO, Tretiakova M, Jagadeeswaran S, Montag A, Becker A, Kenny HA, et al: Loss of E-cadherin promotes ovarian cancer metastasis via alpha 5-integrin, which is a therapeutic target. Cancer Res 68: 2329-2339, 2008

23. Tang B, Peng ZH, Yu PW, Yu G and Qian F: Expression and significance of $\mathrm{Cx} 43$ and E-cadherin in gastric cancer and metastatic lymph nodes. Med Oncol 28: 502-508, 2011.

24. Chao YL, Shepard CR and Wells A: Breast carcinoma cells re-express E-cadherin during mesenchymal to epithelial reverting transition. Mol Cancer 9: 179, 2010. 